\title{
NANOPOROUS SILICA FILMS AS NOVEL BIOMATERIAL: APPLICATIONS IN THE MIDDLE EAR
}

\author{
Behrens $\mathrm{P}^{1}$, Ehlert $\mathrm{N}^{1}$, Müller $\mathrm{PP}^{2}$, Stieve $\mathrm{M}^{3,4}$, Lenarz $\mathrm{T}^{3}$ \\ ${ }^{1}$ Institute for Inorganic Chemistry, Leibniz University Hannover, Germany \\ ${ }^{2}$ Helmholtz Centre for Infection Research, Braunschweig, Germany \\ ${ }^{3}$ Hals-Nasen-Ohren-Klinik, Medizinische Hochschule Hannover, Germany \\ ${ }^{4}$ new address: Klinik für Hals-, Nasen- und Ohrenheilkunde, Universitätsklinikum Schleswig-Holstein, Germany \\ peter.behrens@acb.uni-hannover.de
}

\begin{abstract}
We have introduced nanoporous silica as a novel biomaterial. Nanoporous silica is non-toxic and biocompatible. It provides a high surface area and pore volume, uniform and tunable pore sizes and the possibility for chemical modification. We have shown that nanoporous silica coatings on middle ear prostheses provide a suitable basis for installing various functionalizations which can improve the healing after the insertion of the implant.
\end{abstract}

Keywords: Nanoporous silica, middle ear prosthesis, drug delivery, growth factors

\section{Introduction}

Nanoporous silica materials, discovered in the early 1990 s, have aroused great interest for a variety of applications. The most important properties of these materials are their high surface areas and pore volumes, the tunable and uniform pore size, and the convenience with which chemical modifications can be carried out due to the presence of surface silanol groups. Nanoporous silicas meet the basic requirements for biomaterials, as they are non-toxic and have a high biocompatibility. Usual synthetic procedures for nanoporous silicas deliver a powder of aggregated particles which is difficult to apply in biomedical devices. A lot of research has been performed on nanoporous silica nanoparticles. An alternative application form are coatings of nanoporous silica, which can be applied directly onto a pre-formed implant or prosthesis. Thin films of nanoporous silica can be produced on various substrates using dip- or spin-coating procedures. Considering the possibility to directly equip implants with the favourable properties of nanoporous silica, we have established coatings of this material on known implant materials, on the use of the possible functions of such coatings and on testing them in cell culture and animal experiments.

\section{Methods}

Nanoporous silica materials are created by a condensation reaction of amorphous silica around ordered aggregates of amphiphilic molecules. These molecules are then removed by a calcination or extraction step leading to pores with diameters from 2 to $10 \mathrm{~nm}$ with various pore topologies like cubic or hexagonal in $2 \mathrm{D}$ or $3 \mathrm{D}$. The materials have been characterized by XRD, scanning electron microscopy, porosimetry and other methods.

\section{Results}

We have shown by cell-culture and animal experiments that nanoporous silica applied as a coating on total ossicular replacement prostheses made from Bioverit ${ }^{\circledR}$ II are perfectly biocompatible. A local drug delivery system releasing the antibiotic ciprofloxacin was established. Its functioning was established by effectively combating an intentionally placed infection of Pseudomonas aerugino$s a$. Furthermore, the bone growth factor BMP2 could be immobilized on the nanoporous coating under preservation of the biological activity of the protein [1].

\section{Discussion}

The use of nanoporous silica coatings on implant surfaces appears offers many possibilities for the equipment of prostheses with various biological and medicinal functions, for example for use in local drug delivery devices or for the attachment of sensitive biomolecules. The results obtained should be easily transferable to other types of prostheses and implants. These results have recently been summarized in a tutorial review [1].

\section{Acknowledgement}

This work was supported within the Collaborative Research Center (Sonderforschungsbereich) of the DFG. We thank all our co-workers in the work package D1 for their enthusiasm and willingness to cooperate; they are named in the references referring to our original papers as given in ref. [1]. This work has also profited from discussions with partners from the Excellence Cluster "Hearing4All".

\section{Bibliography}

[1] Ehlert, N., Mueller, P.P., Stieve, M., Lenarz, T., Behrens, P.: Mesoporous silica films as a novel biomaterial: applications in the middle ear. Chem.Soc. Rev., vol. 42, pp. 3847-3861, 2013. DOI: 10.1039/c3cs35359a 